\title{
Constant-coupling theory of nematic liquid crystals
}

\author{
Ping Sheng and Peter J. Wojtowicz \\ RCA Laboratories, Princeton, New Jersey 08540 \\ (Received 4 June 1976)
}

\begin{abstract}
The well-known constant-coupling theory of ferromagnetism has been adapted to the problem of the nematic liquid crystal. A cluster variational formulation of the theory is developed. The calculations are based on an effective pair potential which is derived from the most general form of interaction by taking suitable averages. Expressions for the long-range order, short-range order, energy, and free energy are derived. Numerical calculations have been performed for a variety of effective interaction potentials. The temperature dependences of the order parameters and thermodynamic functions have been determined. As required by experiment, the theory is found to display a first-order nematic-isotropic phase transition. The results of the present theory are compared to those of previous calculations; questions concerning the validity of the mean-field theory have been clarified. The numerical results are also compared to a variety of experimental data.
\end{abstract}

\section{INTRODUCTION}

The statistical mechanics of nematic liquid crystals is not nearly as advanced as the corresponding theories of other ordered phases. The statistical mechanics of the various magnetic phases and of their critical behavior, for example, is very highly developed. For nematic liquids on the other hand, the only theories available include the hardrod model, ${ }^{1,2}$ various forms of the mean-field approximation,,$^{3-7}$ and some Monte Carlo calculations. ${ }^{8,9}$ In this paper we describe a new statistical treatment of the nematic phase based on the well known "constant-coupling" theory of ferromagnets.

The constant-coupling theory was initially introduced by Kasteleijn and Van Kranendonk ${ }^{10}$ to provide an improvement over the Weiss mean-field models. The precise relationship of this theory to the rigorous cluster expansion treatment of ferromagnets was subsequently demonstrated by Strieb, Callen, and Horwitz. ${ }^{11}$ Numerous applications of the theory to problems in ferromagnetism and antiferromagnetism have appeared, ${ }^{12-15}$ and in all cases the results are found to be more representative of real magnets than those given by the mean-field approximation. The constantcoupling treatment provides more accurate estimates of the critical points, gives a more realistic temperature dependence of the magnetization, heat capacity, and high-temperature susceptibility, and is capable of accounting for many of the effects of short-range order.

With its basis in statistical mechanics thus firmly established, the constant-coupling theory appears to be a natural candidate for the extension of the theory of nematic liquid crystals beyond the mean-field approximation. In Sec. II we will develop a cluster variational approach to the constant-coupling theory of the nematic phase by fol- lowing the formulation of this method given by Smart. ${ }^{16}$ The effective pair interaction potential used is derived from a general form of intermolecular pair potential by taking suitable averages. Expressions for the long-range order, short-range order, energy, and free energy are then derived and the self-consistency of the theory is explored.

In Sec. III we describe the results of the theory obtained by numerical solution of the equations for a variety of effective interaction potentials. The temperature dependence of the order parameters and thermodynamic quantities are of special interest. As required by experiment, the theory is found to display a first-order nematic to isotropic liquid-phase transition.

Section IV is devoted to a comparison of the results of this theory to those of the mean-field treatment, the Monte Carlo calculations, and several other models. The difficulties encountered in other attempts ${ }^{17,18}$ at the cluster variational approach are also examined and resolved. We conclude this paper in Sec. $\mathrm{V}$ by comparing our numerical results to experiments on some real nematic materials.

\section{FORMULATION}
A. Pair-interaction potential

The most general form of pair-interaction potential $v_{12}$ between two axially symmetric molecules can be written in the form of the Pople expansion $^{19}$

$$
\begin{aligned}
& v_{12}\left(r, \theta_{1}^{\prime}, \varphi_{1}^{\prime}, \theta_{2}^{\prime}, \varphi_{2}^{\prime}\right) \\
& =4 \pi \sum_{L_{1}=0}^{\infty} \sum_{L_{2}=0}^{\infty} \sum_{m} u_{L_{1} L_{2}, m}(r) \\
& \quad \times Y_{L_{1} m}\left(\theta_{1}^{\prime}, \varphi_{1}^{\prime}\right) Y_{L_{2} m}^{*}\left(\theta_{2}^{\prime}, \varphi_{2}^{\prime}\right) .
\end{aligned}
$$


Here $r$ is the distance of separation between the centers of the two molecules; $\theta_{1}^{\prime}, \theta_{2}^{\prime}, \varphi_{1}^{\prime}, \varphi_{2}^{\prime}$ are the polar angles of the two molecules defined as shown in Fig. $1 ; Y_{L m}(\theta, \varphi)$ is the usual spherical harmonic; * denotes complex conjugation; and $u_{L_{1} L_{2}, m}(r)$ are functions of $r$ that vanish for $|m|>L_{1}, L_{2}$. Since $v_{12}$ is a real and even function of $\varphi_{1}^{\prime}-\varphi_{2}^{\prime}$, the functions $u_{L_{1} L_{2}, m}(r)$ have the property that $u_{L_{1} L_{2}, m}(r)$ $=u_{L_{1} L_{2},-m}(r)$.

A rigorous theory of a fluid system based on the general form of pairwise interaction potential of Eq. (1) is impossibly difficult. A simpler approach is to derive an "effective" pairwise interaction potential which can be obtained from Eq. (1) by performing certain averages. That "effective" pairwise interaction potential will then be the starting point of the theory presented in this paper.

In order to derive an effective interaction potential, we first express $v_{12}$ in terms of a polar coordinate system based on the director of the nematic phase $\hat{n}$ as the polar axis. The coordinate axes for the molecules 1 and 2 must be rotated from that shown in Fig. 1(a) to that shown in Fig. 1(b). The unprimed angles now describe the orientations of the molecules with respect to the new rotated coordinate system. In the new coordinate system $v_{12}$ has the form

$$
\begin{aligned}
v_{12}=4 \pi \sum_{L_{1}=0}^{\infty} \sum_{L_{2}=0}^{\infty} \sum_{m} \sum_{p, q} & u_{L_{1} L_{2}, m}(r)\left(D_{p m}^{L_{1}}\right) \\
& \times Y_{L_{1} p}\left(\theta_{1}, \varphi_{1}\right)\left(D_{q m}^{L_{2}}\right)^{*} \\
& \times Y_{L_{2} q}^{*}\left(\theta_{2}, \varphi_{2}\right)
\end{aligned}
$$

where the $D_{p m}^{L}$ are the elements of the Wigner rotation matrices. ${ }^{20}$ Now we take two successive averages of $v_{12}$. First, we average over all orientations of the intermolecular vector $\overrightarrow{\mathbf{r}}$. Next, we average $v_{12}$ over all values of the intermolecular separation $r$. The combination of these two averages will remove all dependences of $v_{12}$ on the intermolecular vector $\vec{r}$. Such a double average

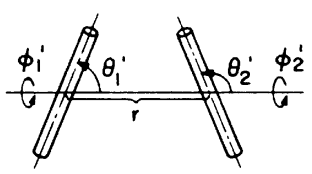

(a)

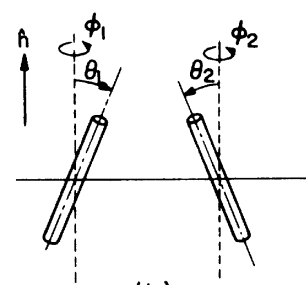

(b)
FIG. 1. Coordinate systems required to describe the interaction between two axially symmetric molecules: (a) the intermolecular vector $r$ is the mutual polar axis and (b) the director $n$ is the polar axis for each molecule. (denoted by $\langle\langle\rangle\rangle$ ) has been carried out by Humphries et $a l .{ }^{6}$ The resulting effective interaction potential $V_{12}$ can be expressed as a series in Legendre polynomials $P_{L}$ :

$$
V_{12} \equiv\left\langle\left\langle v_{12}\right\rangle\right\rangle=\sum_{L} C_{L} P_{L}\left(\cos \theta_{12}\right) .
$$

Here $C_{L}$ 's are constants, and $\theta_{12}$ is the relative angle between the long axes of molecules 1 and 2 . In the following we will assume that $C_{2}<0$ and that the interaction potential $V_{12}$ cannot distinguish between the heads and tails of the molecules, which implies that only the even $L$ terms will be included in the sum. If we further define $v_{0} \equiv\left|C_{2}\right|, \alpha_{L} \equiv C_{L} /$ $C_{2}$, then $V_{12}$ can be put in the form

$$
V_{12}=-v_{0} \sum_{L(\text { even })} \alpha_{L} P_{L}\left(\cos \theta_{12}\right) .
$$

It should be noted that $\alpha_{2} \equiv 1$ and $v_{0}>0$ by definition.

\section{B. Cluster variational approach}

Our task now is the formulation of a theory so that the thermodynamic properties of the system can be calculated from $V_{12}$. The simplest approach is the mean-field theory, which would further reduce $V_{12}$ into a one-particle potential (denoted as $\bar{V}_{12}$ ) by averaging over all possible orientations of molecule 2 . Since the singleparticle orientational distribution is expected to be axially symmetric about the director $\hat{n}$ and therefore independent of $\varphi$, such an average yields

$$
V_{\mathrm{MF}} \equiv \bar{V}_{12}=-v_{0} \sum_{L(\text { even })} \alpha_{L}\left\langle P_{L}\right\rangle P_{L}\left(\cos \theta_{1}\right),
$$

where $\theta_{1}$ is the angle of molecule 1 with respect to the director $\hat{n}$; and $\left\langle P_{L}\right\rangle$, the average value of $P_{L}$, can be regarded as the order parameters of the problem. If each molecule has $z$ neighbors, then the total mean field felt by the molecule is $z V_{\mathrm{MF}}$. Given the form of $V_{\mathrm{MF}}$ as in Eq. (5), the order parameters $\left\langle P_{L}\right\rangle$ can be determined by the self-consistent equations

$$
\left\langle P_{L}\right\rangle=\frac{\int_{0}^{1} P_{L}\left(\cos \theta_{1}\right) \exp \left[-\beta z V_{\mathrm{MF}}\left(\cos \theta_{1}\right)\right] d\left(\cos \theta_{1}\right)}{\int_{0}^{1} \exp \left[-\beta z V_{\mathrm{MF}}\left(\cos \theta_{1}\right)\right] d\left(\cos \theta_{1}\right)},
$$

where $\beta \equiv 1 / k T, k$ being Boltzmann's constant and $T$ the temperature.

In another development, Oguchi ${ }^{21}$ has invented a method in which a pair of molecules interacting with $V_{12}$ is placed in an effective mean field of its neighbors. If each molecule has $z$ neighbors, then the total interaction energy of the pair is given by 


$$
\begin{gathered}
V_{\text {Oguchi }}=(z-1)\left[V_{\mathrm{MF}}\left(\cos \theta_{1}\right)+V_{\mathrm{MF}}\left(\cos \theta_{2}\right)\right]+V_{12} \\
=-v_{0} \sum_{L \text { (even) }} \alpha_{L}\left\{(z-1)\left\langle P_{L}\right\rangle\right. \\
\times\left[P_{L}\left(\cos \theta_{1}\right)+P_{L}\left(\cos \theta_{2}\right)\right] \\
\left.+P_{L}\left(\cos \theta_{12}\right)\right\}
\end{gathered}
$$

where $\cos \theta_{12}=\cos \theta_{1} \cos \theta_{2}+\sin \theta_{1} \sin \theta_{2} \cos \left(\varphi_{1}-\varphi_{2}\right)$. In the Oguchi method the long-range-order parameters $\left\langle P_{L}\right\rangle$ are determined by the consistency conditions

$\left\langle P_{L}\right\rangle=\frac{\int_{0}^{1} \int_{0}^{1} \int_{0}^{1} P_{L}(x) \exp \left(-\beta V_{\text {Oguchi }}\right) d \xi d x d y}{\int_{0}^{1} \int_{0}^{1} \int_{0}^{1} \exp \left(-\beta V_{\text {Oguchi }}\right) d \xi d x d y}$,

where $x \equiv \cos \theta_{1}, y \equiv \cos \theta_{2}$, and $\xi \equiv\left(\varphi_{1}-\varphi_{2}\right) / 2 \pi$. The Oguchi method also yields short-range-order parameters $\tau_{L}$ :

$$
\begin{aligned}
\tilde{V}_{\text {Oguchi }} & =(z-1)\left[\tilde{V}_{\mathrm{MF}}\left(\cos \theta_{1}\right)+\tilde{V}_{\mathrm{MF}}\left(\cos \theta_{2}\right)\right]+V_{12} \\
& =-v_{0} \sum_{L(\text { even })}(z-1) \sigma_{L}\left[P_{L}\left(\cos \theta_{1}\right)+P_{L}\left(\cos \theta_{2}\right)\right]+\alpha_{L} P_{L}\left(\cos \theta_{12}\right) .
\end{aligned}
$$

$\left\langle P_{L}\right\rangle$ can then be determined by using Eq. (8) with $V_{\text {Oguchi }}$ replaced by $\tilde{V}_{\text {Oguchi }}$. Following Smart, ${ }^{16}$ the condition that we are going to impose on the $\sigma$ 's is that the two ways of determining $\left\langle P_{L}\right\rangle$ have to yield identical results. That is,

$$
\begin{aligned}
& \int_{0}^{1} P_{L}(x) \exp \left(\frac{v_{0}}{k T} z \sum_{L \text { (even) }} \sigma_{L} P_{L}(x)\right) d x / \int_{0}^{1} \exp \left(\frac{v_{0}}{k T} z \sum_{L \text { (even) }} \sigma_{L} P_{L}(x)\right) d x \\
&=\int_{0}^{1} \int_{0}^{1} \int_{0}^{1} P_{L}(x) \exp \left[\frac{v_{0}}{k T}\left((z-1) \sum_{L \text { (even) }} \sigma_{L}\left[P_{L}(x)+P_{L}(y)\right]+\sum_{L \text { (even) }} \alpha_{L} P_{L}\left(\cos \theta_{12}\right)\right)\right] d \xi d x d y \\
& \times\left\{\int_{0}^{1} \int_{0}^{1} \int_{0}^{1} \exp \left[\frac{v_{0}}{k T}\left((z-1) \sum_{L \text { (even) }} \sigma_{L}\left[P_{L}(x)+P_{L}(y)\right]+\sum_{L \text { (even) }} \alpha_{L} P_{L}\left(\cos \theta_{12}\right)\right)\right] d \xi d x d y\right\}^{-1} .
\end{aligned}
$$

Here $\cos \theta_{12}=x y+\left[\left(1-x^{2}\right)\left(1-y^{2}\right)\right]^{1 / 2} \cos 2 \pi \xi$. Equation (12) is the heart of the cluster variational approach. There is one equation for every even $L$. In every one of these equations $z$ and $\alpha_{L}$ 's are the inputs and $\sigma_{L}$ 's are the unknowns. Simultaneous solution of all the equations yields the dependence of $\sigma_{L}$ 's on the reduced temperature $k T / v_{0}$. Once the $\sigma_{L}$ are known, the long-range-order parameters, $\left\langle P_{L}\right\rangle$, and the short-range-order parameters, $\tau_{L}$, can easily be determined in the same manner as in the Oguchi method. Namely, we can use Eqs. (8) and (9) with $V_{\text {Oguchi }}$ replaced by $\tilde{V}_{\text {Oguchi }}$.

\section{Statistical thermodynamics}

In order to determine the internal energy of the system in which the pairwise interaction between the molecules is given by $V_{12}$, we note that the internal energy for a single pair $E_{p}$ is given by

$$
E_{p}=-v_{0} \sum_{L(\text { even })} \alpha_{L}\left\langle P_{L}\left(\cos \theta_{12}\right)\right\rangle=-v_{0} \sum_{L(\text { even })} \alpha_{L} \tau_{L} .
$$

Here \langle\rangle denotes thermodynamic averaging. If each molecule has $z$ neighbors, then for each molecule we count $z$ pairs of interactions. Therefore, the total internal energy $E$ of the system is given by

$$
E=\frac{1}{2} N z E_{p}=-\frac{1}{2} v_{0} N z \sum_{L \text { (even) }} \alpha_{L} \tau_{L}
$$

where $N$ is the total number of molecules and the factor $\frac{1}{2}$ arises because each interaction has been 
counted twice.

Next, we write down the free energy $F$ of the system. The free-energy function must satisfy two requirements: (i) Setting $\partial F / \partial \sigma_{L}=0$ should give Eq. (12), the consistency condition for the $\sigma_{L}$. (ii) $\partial(\beta F) / \partial \beta$, evaluated at those values of $\sigma_{L}$ which satisfy Eq. (12), should yield $E$. The first require- ment follows from the fact that the solutions determined from Eq. (12) must be those that represent the extrema of free energy. The second requirement follows directly from thermodynamics. It is easily verified that a free-energy function which satisfies these two requirements can be written

$$
\begin{aligned}
\frac{F}{N k T}= & (z-1) \ln \left[\int_{0}^{1} \exp \left(\frac{v_{0}}{k T} z \sum_{L \text { (even) }} \sigma_{L} P_{L}(x)\right) d x\right] \\
& -\frac{z}{2} \ln \left\{\int_{0}^{1} \int_{0}^{1} \int_{0}^{1} \exp \left[\frac{v_{0}}{k T}\left((z-1) \sum_{L \text { (even) }} \sigma_{L}\left[P_{L}(x)+P_{L}(y)\right]+\sum_{L(\text { even })} \alpha_{L} P_{L}\left(\cos \theta_{12}\right)\right)\right] d \xi d x d y\right\}+\text { const. }
\end{aligned}
$$

The question arises as to whether there are other free-energy functions which satisfy the same two requirements. In other words, are the two requirements stated above sufficient to uniquely determine the free-energy function? In the appendix we show that the two conditions are only sufficient to determine (to within a constant) the free energy $\beta F$ at those values of $\sigma_{L}$ 's which satisfy Eq. (12), denoted as $\sigma_{L}^{0}(T)$. Therefore, the answer to the uniqueness question is that up to a constant, the values of $\beta F$ as given by Eq. (15) is unique over the domain of $\sigma_{L}^{0}(T)$.

\section{NUMERICAL CALCULATIONS}

In this section we present results of numerical calculations based on an interaction potential in which only the leading term of $V_{12}$ is retained. Namely, we will let

$$
V_{12}=-v_{0} P_{2}\left(\cos \theta_{12}\right) \text {. }
$$

The effect of including one higher-order term, $P_{4}\left(\cos \theta_{12}\right)$, in $V_{12}$ will also be investigated.

\section{A. Thermodynamic properties}

Theoretically, $V_{12}$ given by Eq. (16) can be directly substituted into Eq. (12). The resulting equations will have $\alpha_{2}=1$ and all the other $\alpha_{L}$ 's equal to zero. The values of $\sigma_{L}$ 's at each reduced temperature $k T / v_{0}$ can then be obtained by the simultaneous solution of all the equations. However, from the mean-field theory it is expected that if $V_{12}$ is of the form expressed by Eq. (16), then the mean field experienced by molecule 1 would mostly be of the form $P_{2}\left(\cos \theta_{1}\right)$. That is, $\sigma_{2}$ is expected to be much larger than all the other $\sigma_{L}$ 's. This expectation is borne out by calculations as shown in a later section. Therefore, for simplicity we will retain only $\sigma_{2}$ and $\sigma_{4}$ in Eq. (12) and set all the other $\sigma_{L}$ 's equal to zero. In this manner we have reduced the problem to two simultaneous equations for the determination of $\sigma_{2}$ and $\sigma_{4}:$

$$
\begin{aligned}
& \int_{0}^{1} P_{2}(x) f_{1}(x) d x \\
& \quad=\int_{0}^{1} \int_{0}^{1} \int_{0}^{1} P_{2}(x) f_{2}(x, y, \xi) d x d y d \xi, \\
& \int_{0}^{1} P_{4}(x) f_{1}(x) d x \\
& =\int_{0}^{1} \int_{0}^{1} \int_{0}^{1} P_{4}(x) f_{2}(x, y, \xi) d x d y d \xi,
\end{aligned}
$$

where

$$
\begin{aligned}
& f_{1}(x)=\frac{\exp \left\{\left(v_{0} / k T\right) z\left[\sigma_{2} P_{2}(x)+\sigma_{4} P_{4}(x)\right]\right\}}{\int_{0}^{1} \exp \left\{\left(v_{0} / k T\right) z\left[\sigma_{2} P_{2}(x)+\sigma_{4} P_{4}(x)\right]\right\} d x}, \\
& f_{2}(x, y, \xi)=\frac{\exp \left\{\left(v_{0} / k T\right)\left[(z-1)\left[\sigma_{2} P_{2}(x)+\sigma_{2} P_{2}(y)+\sigma_{4} P_{4}(x)+\sigma_{4} P_{4}(y)\right]+P_{2}\left(\cos \theta_{12}\right)\right]\right\}}{\int_{0}^{1} \int_{0}^{1} \int_{0}^{1} \exp \left\{\left(v_{0} / k T\right)\left[(z-1)\left[\sigma_{2} P_{2}(x)+\sigma_{2} P_{2}(y)+\sigma_{4} P_{4}(x)+\sigma_{4} P_{4}(y)\right]+P_{2}\left(\cos \theta_{12}\right)\right]\right\} d x d y d \xi} .
\end{aligned}
$$

The solution of Eqs. (17a) and (17b) for a given $z$ yields values of $\sigma_{2}$ and $\sigma_{4}$ at each reduced temperature $k T / v_{0}$. Once these values are known, we can calculate the long-range-order parameters

$$
\begin{aligned}
& \left\langle P_{2}\right\rangle \text { and }\left\langle P_{4}\right\rangle \text { : } \\
& \left\langle P_{2,4}\right\rangle=\int_{0}^{1} P_{2,4}(x) f_{1}(x) d x .
\end{aligned}
$$


The short-range order $\tau_{2}$ is obtained similarly,

$$
\tau_{2}=\int_{0}^{1} \int_{0}^{1} \int_{0}^{1} P_{2}\left(\cos \theta_{12}\right) f_{2}(x, y, \xi) d x d y d \xi .
$$

The free energy can be obtained by using Eq. (15), which can be cast in the form

$$
\begin{array}{r}
\frac{F}{N}=-\frac{1}{2} v_{0} z \tau_{2}-k T \\
\left((z-1) \int_{0}^{1} f_{1}(x) \ln f_{1}(x) d x\right. \\
-\frac{z}{2} \int_{0}^{1} \int_{0}^{1} \int_{0}^{1} f_{2}(x, y, \xi) \\
\left.\times \ln f_{2}(x, y, \xi) d x d y d \xi\right)
\end{array}
$$

+ const.

where the first term is the internal energy per particle and the second term is the entropy contribution to the free energy.

It should be noted that $\sigma_{2}, \sigma_{4}=0$ is a solution of Eqs. (17a) and (17b) for any value of $k T / v_{0}$. This is the disordered phase, the isotropic liquid. At large values of $k T / v_{0}$ this is the only solution. However, below a certain reduced temperature two more solutions appear. The equilibrium solution in that temperature range is then determined by the criterion of minimum free energy. The calculation is done on a computer using Gaussian integration techniques and Newton's method in locating the solutions. In Fig. 2 we present the variation of $\left\langle P_{2}\right\rangle$ and $\tau_{2}$ vs $k T / v_{0}$ for $z=6$. The accurate values of $\left\langle P_{2}\right\rangle$ and $\tau_{2}$ at the transition, the transition temperature, and the entropy discontinuity $\Delta S$ are given in Table I for $z=10,8,6,4$. The results for $z=\infty$, the mean-field theory, are also presented for comparison. Since in liquid crystals $z$ is the "average number" of nearest

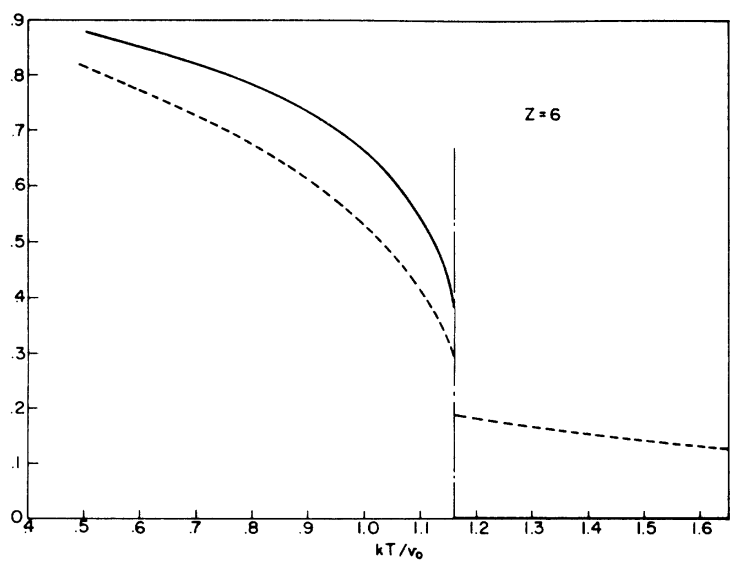

FIG. 2. Temperature variation of long- and shortrange order for $z=6 .\left\langle P_{2}\right\rangle$ is the solid curve while $\tau_{2}$ is the dashed curve.
TABLE I. Constant-coupling theory results using the potential of Eq. (16). $\left\langle P_{2}\right\rangle_{c}$ is the long-range-order parameter at the transition temperature $T_{c}$, while $\tau_{2}^{(n)}$ and $\tau_{2}^{(1)}$ are the short-range-order parameters of the nematic and isotropic phases, respectively, at $T_{c} . \Delta S$ is the entropy change at the transition. The column for $z=\infty$ represents the results of the mean-field theory (Ref. 4).

\begin{tabular}{lccccc}
\hline \hline \multicolumn{1}{c}{$z$} & 4 & 6 & 8 & 10 & $\infty$ \\
\hline$\left\langle P_{2}\right\rangle_{c}$ & 0.356 & 0.382 & 0.399 & 0.405 & 0.429 \\
$\tau_{2}^{(n)}$ & 0.393 & 0.298 & 0.264 & 0.245 & $\ldots$ \\
$\tau_{2}^{(i)}$ & 0.320 & 0.189 & 0.134 & 0.103 & $\ldots$ \\
$k T_{c} / z v_{0}$ & 0.1740 & 0.1933 & 0.2011 & 0.2055 & 0.2202 \\
$\Delta S / N$ & 0.210 & 0.282 & 0.323 & 0.346 & 0.418 \\
\hline \hline
\end{tabular}

neighbors, $z$ can take on nonintegral values. In Figs. 3 and 4 we show the variation of $k T_{c} / v_{0}$ and the transition entropy $\Delta S / N k$ as a function of $z$, treated here as a continuous variable. It should be noted that as a function of $z$, the nematicisotropic phase transition tends to be more "second-order-like" as $z$ decreases. More specifically, the discontinuities in $\left\langle P_{2}\right\rangle$ and $\tau_{2}$ at the transition become smaller, and $\Delta S$ decreases rapidly as $z$ decreases. The vanishing of transi-

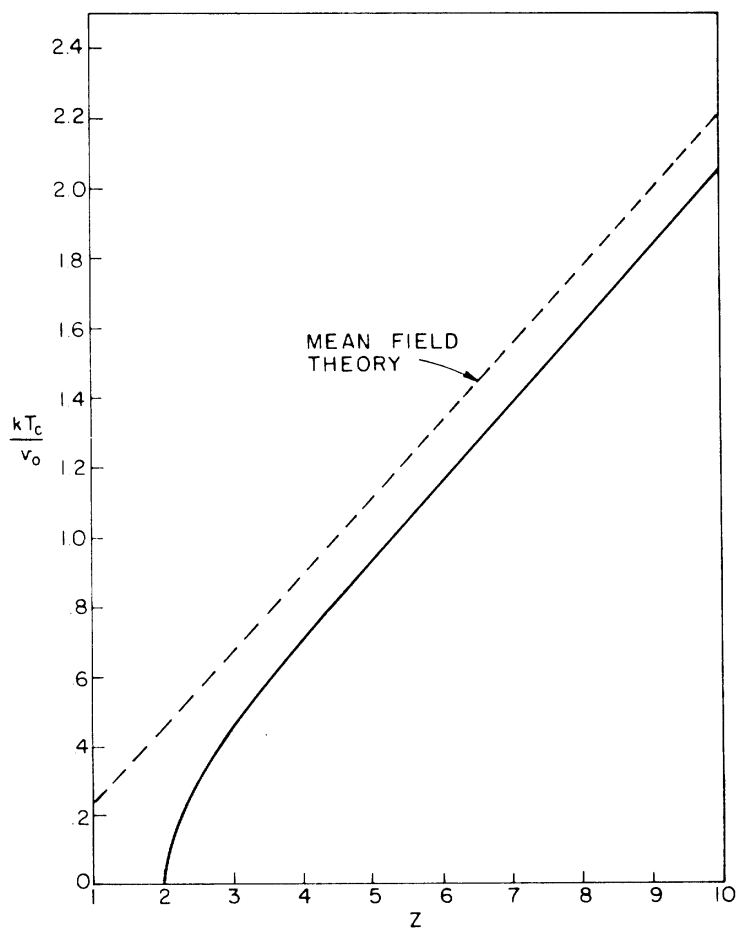

FIG. 3. Dependence of the first-order phase transition temperature $T_{c}$ on effective nearest-neighbor number $z$. 


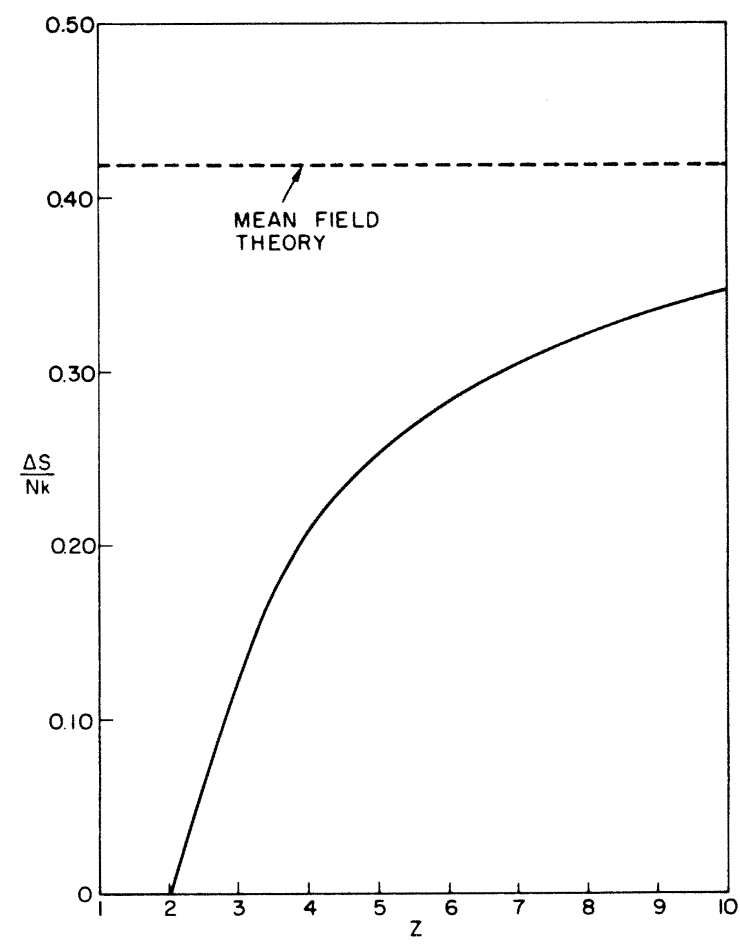

FIG. 4. Dependence of the entropy discontinuity $\Delta S$ on effective nearest-neighbor number $z$.

tion temperature at $z=2$ is understandable since the system has to be one-dimensional for $z=2$, and it is a well-known fact that one-dimensional systems cannot have phase transitions at finite temperatures. In Fig. 5 we show the variation of the specific heat $C$,

$$
\frac{C}{N k}=-\frac{z}{2} \frac{d \tau_{2}}{d\left(k T / v_{0}\right)},
$$

as a function of temperature for $z=6$.

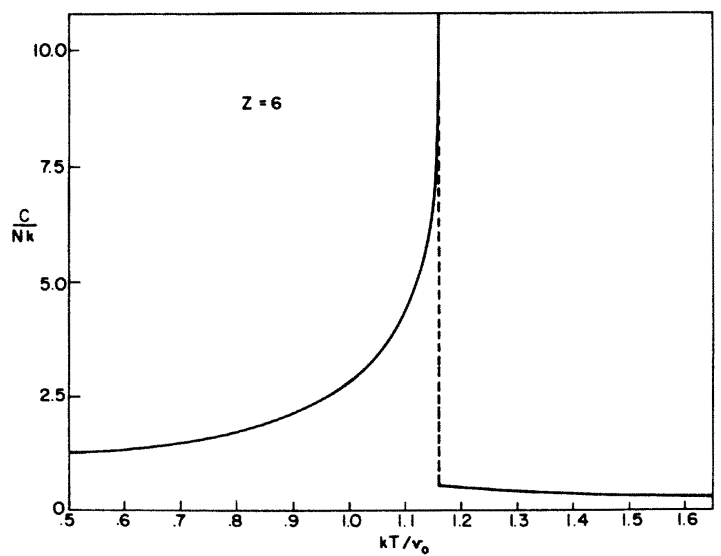

FIG. 5. Temperature variation of the heat capacity for $z=6$.

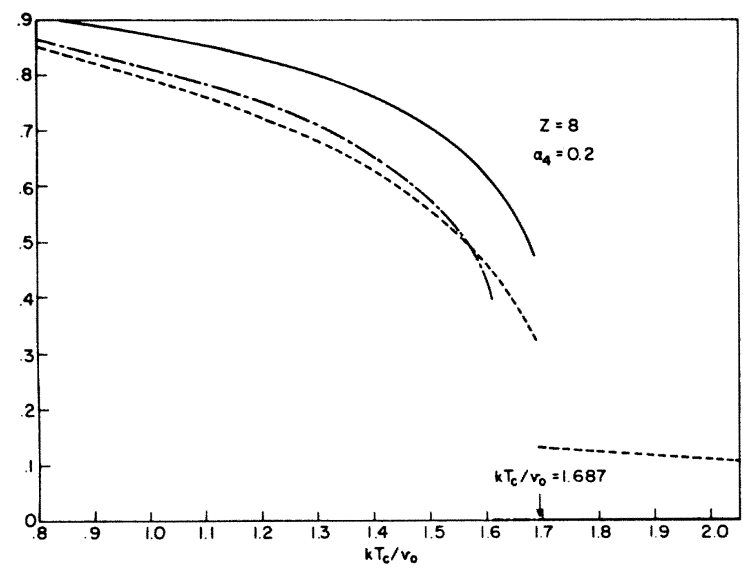

FIG. 6. Temperature variation of the long- and shortrange order for $z=8$ with $\alpha_{4}=+0.2 .\left\langle P_{2}\right\rangle$ is the solid curve while $\tau_{2}$ is the dashed curve. The dot-dashed curve is $\left\langle P_{2}\right\rangle$ for $z=8$ and $\alpha_{4}=0$ for comparison.

\section{B. Effect of the $\boldsymbol{P}_{4}$ term in intermolecular interaction}

The calculation mentioned above can easily be adapted for a $V_{12}$ of the form

$$
V_{12}=-v_{0}\left[P_{2}\left(\cos \theta_{12}\right)+\alpha_{4} P_{4}\left(\cos \theta_{12}\right)\right] \text {. }
$$

We replace $P_{2}\left(\cos \theta_{12}\right)$ in Eq. (18b) by $P_{2}\left(\cos \theta_{12}\right)$ $+\alpha_{4} P_{4}\left(\cos \theta_{12}\right)$. Then the solution of Eqs. (17a) and (17b) yields values of $\sigma_{2}$ and $\sigma_{4}$ at every $k T / v_{0}$. In Figs. 6 and 7 we show $\left\langle P_{2}\right\rangle$ and $\tau_{2}$ as function of $k T / v_{0}$ for $z=8$ and $\alpha_{4}= \pm 0.2$. It is seen that the values of transition temperature, $\left\langle P_{2}\right\rangle$, and $\tau_{2}$ are all raised for $\alpha_{4}=0.2$ and are depressed for $\alpha_{4}$ $=-0.2$. $\left\langle P_{2}\right\rangle$ for $\alpha_{4}=0$ is also plotted for comparison.

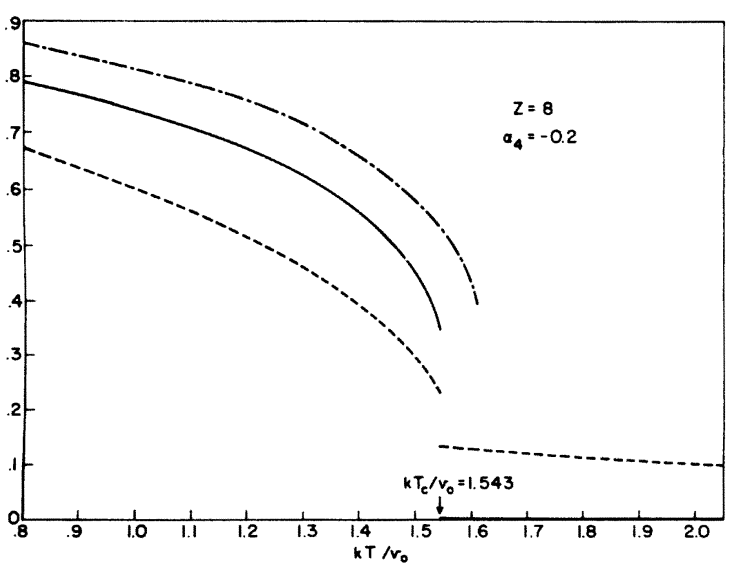

FIG. 7. Temperature variation of the long- and shortrange order for $z=8$ with $\alpha_{4}=-0.2 .\left\langle P_{2}\right\rangle$ is the solid curve while $\tau_{2}$ is the dashed curve. The dot-dashed curve is $\left\langle P_{2}\right\rangle$ for $z=8$ and $\alpha_{4}=0$ for comparison. 


\section{Paranematic susceptibility}

When a magnetic field is applied to a nematic liquid crystal in its isotropic phase, a small amount of nematic order will be induced due to the diamagnetic anisotropy of liquid crystal molecules. The amount of order is proportional to the square of the magnetic field

$$
\left\langle P_{2}\right\rangle=\eta H^{2},
$$

where the proportionality constant $\eta$ always increases in magnitude as the transition temperature is approached from above. In fact, in the meanfield theory and the Landau-deGennes theory $\eta$ is given as

$$
\eta \propto\left(T-T_{c}^{*}\right)^{-1},
$$

where $T_{c}^{*}$ is a temperature lower then $T_{c}$, the transition temperature, and can be interpreted as the temperature below which the supercooling of the isotropic phase becomes impossible. In the meanfield theory we have the relation

$$
T_{c}^{*}=0.9082 T_{c} .
$$

Experimentally, $T_{c}^{*}$ is about $0.99 T_{c}$. Therefore, there is a wide discrepancy between the meanfield theory and the experimental result. In this section we examine the temperature dependence of $\eta$ and calculate the magnitude of $T_{c}^{*}$ in the constant-coupling theory.

When a magnetic field $H$ is applied, each molecule experiences an orienting potential of the form

$$
W_{H}=-\frac{1}{3} H^{2} \Delta \chi P_{2}(\cos \theta),
$$

where $\theta$ is the angle between the long axis of the molecule and the field direction, and $\Delta \chi$ is the dia- magnetic anisotropy for a single molecule. This potential has to be added to the mean field each molecule experiences from its neighbors. Therefore, in the self-consistency condition, Eq. (12), we have to replace

$$
\frac{v_{0}}{k T} z \sum_{L(\text { even })} \sigma_{L} P_{L}(x)
$$

by

$$
\frac{v_{0}}{k T}\left(z \sum_{L(\text { even })} \sigma_{L} P_{L}(x)+\frac{H^{2} \Delta \chi P_{2}(x)}{3 v_{0}}\right)
$$

on the left-hand side of the equation and replace

$$
\frac{v_{0}}{k T}(z-1) \sum_{L(\text { even })} \sigma_{L}\left[P_{L}(x)+P_{L}(y)\right]
$$

by

$$
\begin{gathered}
\frac{v_{0}}{k T}\left((z-1) \sum_{L(\text { even })} \sigma_{L}\left[P_{L}(x)+P_{L}(y)\right]\right. \\
\left.+\frac{H^{2} \Delta \chi\left[P_{2}(x)+P_{2}(y)\right]}{3 v_{0}}\right)
\end{gathered}
$$

on the right-hand side of the equation. Since the quantity $H^{2} \Delta \chi / v_{0}$ is usually small for $H<10^{6} \mathrm{Oe}$, we can assume that the $\sigma_{L}$ will also be small (since in the limit of $H \rightarrow 0$ all $\sigma_{L} \rightarrow 0$ in the isotropic phase). As a result exponentials of these quantities can be expanded, yielding

$$
\begin{aligned}
\left\langle P_{2}\right\rangle & =\frac{v_{0}}{5 k T}\left(z \sigma_{2}+\frac{H^{2} \Delta \chi}{3 v_{0}}\right) \\
& =\frac{I v_{0}}{2 k T}\left((z-1) \sigma_{2}+\frac{H^{2} \Delta \chi}{3 v_{0}}\right),
\end{aligned}
$$

with

$$
\begin{aligned}
I \equiv \int_{0}^{1} \int_{0}^{1} \int_{0}^{1} & {\left[P_{2}(x)+P_{2}(y)\right]^{2} \exp \left(\frac{v_{0}}{k T} \sum_{L(\text { even })} \alpha_{L} P_{L}\left(\cos \theta_{12}\right)\right) d \xi d x d y } \\
& \times\left[\int_{0}^{1} \int_{0}^{1} \int_{0}^{1} \exp \left(\frac{v_{0}}{k T} \sum_{L(\text { even })} \alpha_{L} P_{L}\left(\cos \theta_{12}\right)\right) d \xi d x d y\right]^{-1}
\end{aligned}
$$

From Eq. (28) it is easily calculated that

$$
\sigma_{2}=\frac{H^{2} \Delta \chi}{3 v_{0}} \frac{5 I-2}{5 I-(5 I-2) z} .
$$

Substitution of Eq. (30) into Eq. (28) yields

$$
\left\langle P_{2}\right\rangle=\frac{\Delta \chi}{15 k T} \frac{5 I}{5 I-(5 I-2) z} H^{2} .
$$

Comparison of Eq. (31) with Eq. (24) gives

$$
\eta=\frac{\Delta \chi}{15 k T}\left[1-\left(\frac{5 I-2}{5 I}\right) z\right]^{-1} .
$$

Since $T_{c}^{*}$ can be defined as that temperature at which $\eta$ diverges, from Eq. (32) we obtain the equation

$$
I\left(T_{c}^{*}\right)=\frac{2}{5} z /(z-1) .
$$

The values of $I$ at various temperatures have been calculated on computer for the case of $V_{12}$ $=-v_{0} P_{2}\left(\cos \theta_{12}\right)$. In Fig. 8 we plot $1 / \eta$ as a function of $k T / v_{0}$ for $z=6$. The curves are extended below $k T_{c} / v_{0}$ for the sake of illustration. It should be noted that $1 / \eta$ is no longer a linear function of $T$, although the deviation from linearity is small. Also, we note that the value of $T_{c}^{*}$ obtained from Eq. (33) is a function of $z$. For $z=6$ we have $T_{c}^{*}$ 


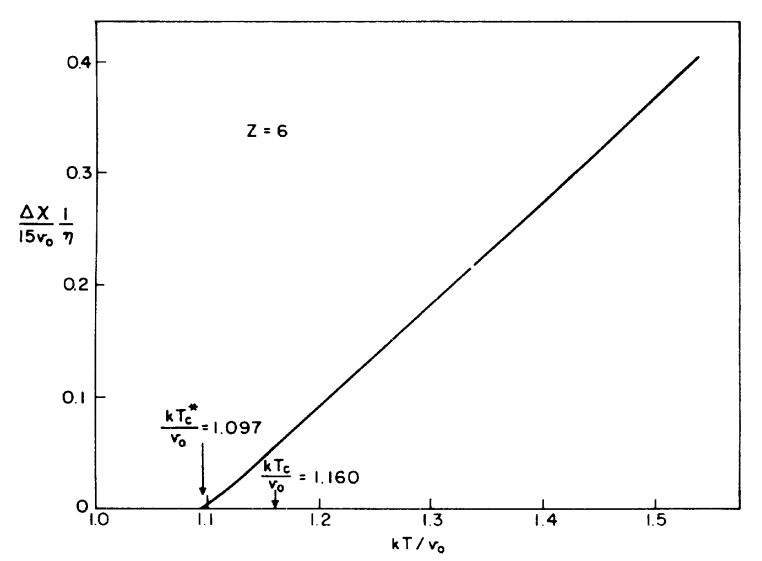

FIG. 8. Temperature variation of the reciprocal paranematic susceptibility $1 / \eta$ for $z=6$.

$=1.097 v_{0} / k=0.9457 T_{c}$ and for $z=8$ we have $T_{c}^{*}$ $=1.510 v_{0} / k=0.9385 T_{c}$. Therefore, in each case the ratio of $T_{c}^{*} / T_{c}$ has been raised above that of the mean-field theory. However, the increase is not enough to explain the experimental result. Similar improvements in $T_{c}^{*} / T_{c}$ have been obtained by Madhusudana and Chandrasekhar ${ }^{22}$ using a somewhat different technique which also includes shortrange-order effects.

\section{Magnitude and the form of the mean field}

It is seen in Sec. II that if $V_{12}=v_{0} P_{2}\left(\cos \theta_{12}\right)$, then, in the mean-field theory, the mean field as experienced by molecule 1 due to molecule 2 is given by

$$
V_{\mathrm{MF}}=-v_{0}\left\langle P_{2}\right\rangle P_{2}\left(\cos \theta_{1}\right) .
$$

Two features of $V_{M F}$ should be noted. First, the magnitude of $V_{\mathrm{MF}}$ is proportional to $\left\langle P_{2}\right\rangle$. Second, the form of $V_{\mathrm{MF}}$ as a function of $\cos \theta_{1}$ is given by $P_{2}\left(\cos \theta_{1}\right)$. In this section we examine the accuracy of these two approximations by using the constantcoupling theory.

In Sec. III A it is shown that in the constantcoupling theory the mean field as experienced by molecule 1 can be approximated by

$$
\tilde{V}_{\mathrm{MF}}=-v_{0}\left[\sigma_{2} P_{2}\left(\cos \theta_{1}\right)+\sigma_{4} P_{4}\left(\cos \theta_{1}\right)\right],
$$

where the values of $\sigma_{2}$ and $\sigma_{4}$ at each reduced temperature $k T / v_{0}$ are determined by Eqs. (17a), (17b), (18a), and (18b). It should be remembered that we have dropped all terms higher than $P_{4}(\cos \theta)$ in the expectation that they are small. In Fig. 9 we show the results of the calculation for $z=8$. It is clear from the graph that $\sigma_{2}$ is not proportional to $\left\langle P_{2}\right\rangle$. The ratio of $\sigma_{2} /\left\langle P_{2}\right\rangle$ is a decreasing function of temperature, reaching the value of $\sim 0.92$ at the transition. We also see that

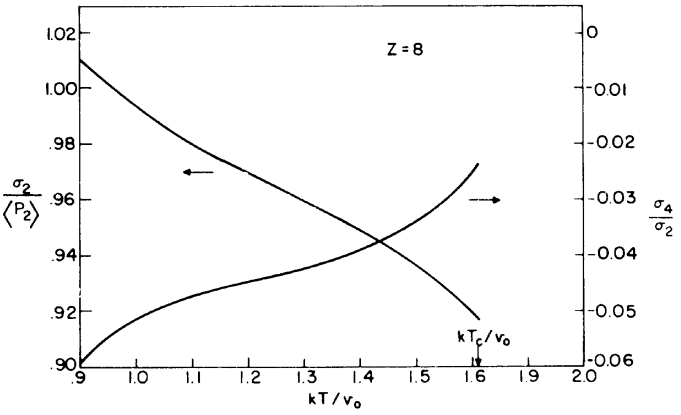

FIG. 9. Temperature variation of the variational parameters $\sigma_{2}$ and $\sigma_{4}$ for $z=8$.

the form of the mean field is not strictly $P_{2}\left(\cos \theta_{1}\right)$ but contains a relatively small portion of $P_{4}\left(\cos \theta_{1}\right)$. $\sigma_{4}$ is always negative, and the absolute magnitude of the ratio $\sigma_{4}$ to $\sigma_{2},\left|\sigma_{4} / \sigma_{2}\right|$, is a decreasing function of temperature, reaching $\sim 0.025$ at the transition. It should be noted that the sharp increase of $\sigma_{2} /\left\langle P_{2}\right\rangle$ and $\left|\sigma_{4} / \sigma_{2}\right|$ below $k T / v_{0}=1.1$ may be caused by the omission of $P_{6}\left(\cos \theta_{1}\right)$ and $P_{8}\left(\cos \theta_{1}\right)$ terms in the series for $\tilde{V}_{\mathrm{MF}}$. However, the figure is indicative of the general trend for these quantities and offers an estimate of the accuracy of the mean-field approximation given by Eq. (34).

Before leaving this section, we point out that in all the numerical calculations the $\alpha_{L}$ and $z$ have been treated as temperature-independent quantities. In the realistic case this assumption may not be valid since in liquid crystals the constituent molecules do not have fixed positions as in a solid, and as a result, the average environment (such as the average number of nearest neighbors) of a molecule may vary with the temperature. In view of such possibilities, the temperature independence of the $\alpha_{L}$ and $z$ should be regarded as a first-order approximation. Further study is required to establish the relationships, if any, between the $\alpha_{L}$ and $z$ on the one hand and $T$, the short-range order (and/or long-range order) on the other.

\section{COMPARISON WITH OTHER THEORIES}

Qualitative and quantitative comparisons of the results of the constant coupling approach with those of the mean-field theory have been given throughout the previous sections. With both methods the nematic-isotropic transformation is found to be a first-order phase transition. The constant coupling theory, however, yields lower values for the transition temperatures, latent heats, entropy changes, and long-range-order parameter discontinuities in all cases. In addition, the present calculation also provides a larger value for the parameter $T_{c}^{*} / T_{c}$.

An improvement over the mean-field theory was 
attempted by Lebwohl and Lasher ${ }^{9}$ using Monte Carlo techniques for the case of $z=6$. A firstorder phase change was observed here also. The critical temperature was found to be $1.124 v_{0} / k$ (in our notation), while the entropy change and long-range-order discontinuity were $0.097 \mathrm{Nk}$ and 0.33 , respectively. The critical temperature compares favorably with our value of $1.160 v_{0} / k$ (Table I). The long-range-order discontinuity compares well with our value of 0.382 and is likewise lower than the mean-field result of 0.429 . The entropy change obtained in the Monte Carlo calculation, however, is much smaller than our value $0.282 N k$. In Sec. V we will see that the experimentally determined entropy changes compare favorably with the constant coupling values.

Raich, Etters, and Flax ${ }^{17}$ and Schultz ${ }^{23}$ have raised the question of whether the first-order phase transition predicted by the mean-field model is merely a spurious result of the mean-field approximation. Based on a cluster variational approach similar to ours but using a separable potential function $\left[V_{12}=V\left(\theta_{1}\right) V\left(\theta_{2}\right)\right.$, where the $\theta_{i}$ give the orientations of the molecules with respect to some external axis], Raich et al. ${ }^{17}$ failed to obtain a phase transition of any kind. With a different kind of statistical model, but again using a separable potential function, Schultz $\mathrm{z}^{23}$ also failed to obtain a phase transition. Priest, ${ }^{18}$ however, has shown that the inability to observe a phase change is caused by the adoption of a separable form of potential. The separable form is unphysical in that it destroys the rotational invariance of the original potential function, and leads to qualitatively different results. Taking a quantized model of a nematic system but using a proper rotationally invariant pair potential, Priest ${ }^{18}$ was able to recover the first-order phase transition.

In this paper we began by assuming a perfectly general pair interaction potential and proceeded to simplify it by making a set of reasonable assumptions which naturally retained the rotational invariance property. Using this simplified potential as the basis for the constant coupling calculations we then obtained first-order phase transitions as required. The results of the meanfield theory, furthermore, can be approached from the present formulation by passing to the limit of very large $z$. We therefore conclude that the firstorder transition of the mean-field theory is not spurious; the theory gives correct qualitative predictions because it does indeed contain the basic elements of the physics of the problem. The situation here is thus no different from the case of magnetism where the relationship of the meanfield model to more general theory is well established.

\section{COMPARISON WITH EXPERIMENT}

Calculations of the entropy discontinuity at the phase change were made in Sec. III A based on the simplest potential function, Eq. (16). The results for $z=4,6,8,10$ are presented in Table I. $\Delta S / N k$ ranges from about 0.2 to 0.35 . Experimental values of $\Delta S / N k$ can be computed from compilations of latent heat and critical temperature data. ${ }^{24}$ Representative values of $\Delta S / N k$ include 4-methoxybenzylidene- $4^{\prime}-n$ butylaniline (MBBA), 0.16 ; 4ethoxybenzylidene- $4^{\prime}-n$ butylaniline (EBBA), 0.29; 4- butoxybenzylidene- $4^{\prime}$ - ethylaniline, 0.15 ; 4cyanobenzylidene- $4^{\prime}-\boldsymbol{n}$-octy loxyaniline (CBOOA), 0.27 ; anisylidene- $p$-aminophenylacetate (APAPA), 0.39 ; anisylidene- $p$-aminophenyl-3-methylvalerate, $0.15-0.3 ; 4-n$-pentyl- $4^{\prime}$ - cyanobiphenyl (PCB), $0.155-0.3 ; 4-n$-nonly-4'-cyanobiphenyl, $0.15-0.5$; $4-n$-pentoxy- $4^{\prime}$ - cyanobiphenyl, $0.15 ; 4-4^{\prime}-\mathrm{di}_{-}$ methoxyazoxybenzene (PAA), $0.12 ; 4-4^{\prime}$-diethoxyazoxybenzene (PAP), 0.34; and $4^{\prime}-4^{\prime}-$ di-nheptyloxyazoxybenzene, $0.25-0.4$. It is seen that the measured values are in good accord with those given by the calculations.

Calculations of the temperature dependence of the long-range-order parameter $\left\langle P_{2}\right\rangle$ were considered in Sec. III A and III B. We have made a comparison of our theoretical results with experimental data ${ }^{25}$ on PAA and PAP using the potential function of Eq. (23). A graphical comparison is shown in Fig. 10. We have arbitrarily chosen $z=6$. The constant $v_{0}$ is then effectively eliminated by expressing both theory and experiment in reduced temperature units, $T / T_{c}$, and forcing the calculated and experimental critical temperatures to agree. This leaves $\alpha_{4}$ as the only remaining parameter to be adjusted. Excellent fits to the data were obtained by choosing $\alpha_{4}=0$ in the case of PAA, and $\alpha_{4}=\frac{1}{3}$

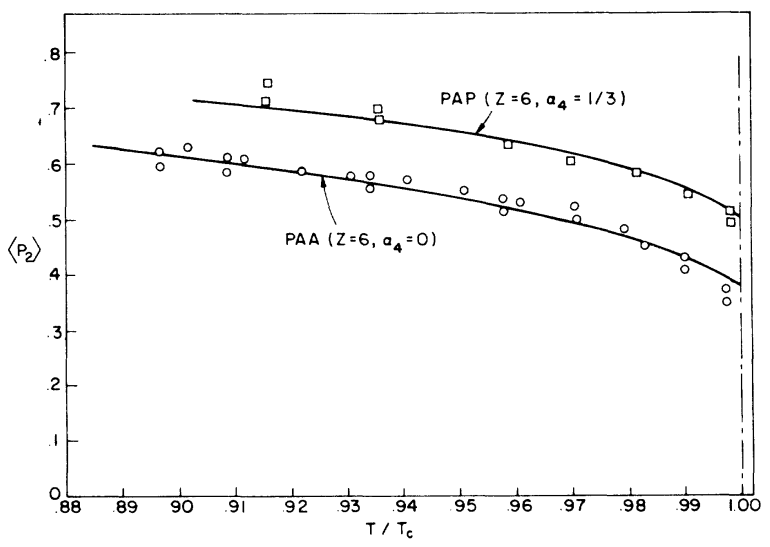

FIG. 10. Comparison of theoretical results and experimental data on the temperature dependence of the long-range order for PAA and PAP. 
in the case of PAP. A more precise fitting could have been made by the simultaneous adjustment of $z$ and $\alpha_{4}$ in a rms procedure and by taking into account the temperature variation of $v_{0}$. We do not feel, however, that such embellishments are instructive considering the approximations already made in arriving at the potential function, Eq. (23).

The most stringent test of any theory of ordered phases is the comparison of calculated and measured heat capacity. Having determined the values of the potential parameters for PAA and PAP from the long-range-order data we can now compute the heat capacities without any further adjustments in the constants. For the potential function, Eq. (23), the heat capacity is obtained from Eq. (14) by differentiation

$$
\frac{C}{N k}=-\frac{z}{2}\left(\frac{d \tau_{2}}{d\left(k T / v_{0}\right)}+\alpha_{4} \frac{d \tau_{4}}{d\left(k T / v_{0}\right)}\right) .
$$

Figures 11 and 12 display the comparisons of theoretical and experimental ${ }^{26}$ orientational heat capacities for PAA and PAP, respectively. The only fitting done for these figures was the establishment of the base line for the experimental points. That is, a roughly constant contribution to the measured heat capacity (representing the nonorientational components) was subtracted from each of the data points. The magnitude of this subtracted portion was chosen so that the lowest temperature data point in each case agrees with the theoretical value. The agreement between theory and experiment is reasonable considering that no further adjustment of potential parameters was made. In particular, the theory is seen to accurately predict the width of the transition region. A more quantitative agreement on the low temperature side of $T_{c}$ could certainly be obtained

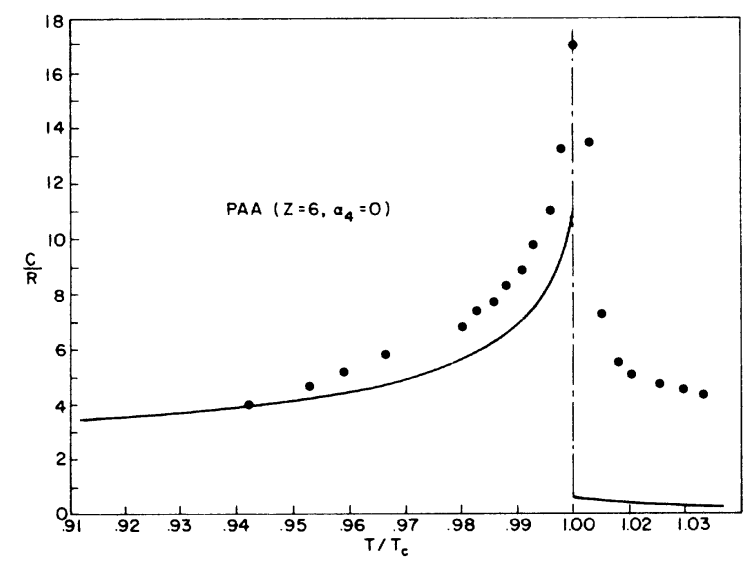

FIG. 11. Comparison of theoretical results and experimental data on the temperature dependence of the orientational heat capacity of PAA.

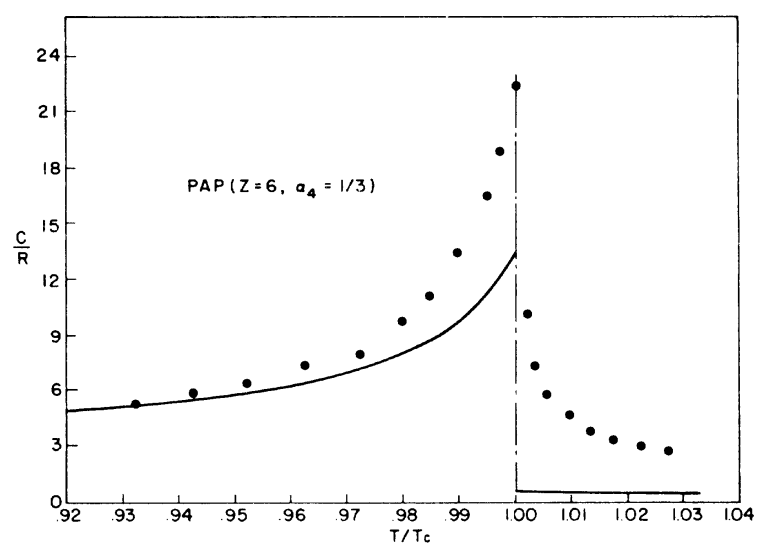

FIG. 12. Comparison of theoretical results and experimental data on the temperature dependence of the orientational heat capacity of PAP.

by simultaneously fitting $\left\langle P_{2}\right\rangle$ and $C$ data through the adjustment of $z$ and $\alpha_{4}$ and taking into account the temperature variation of $v_{0}$. The data on the high-temperature side of $T_{c}$, however, cannot be accounted for quantitatively. As is well known from the example of magnetism, ${ }^{13}$ the constant coupling theory cannot be accurate above the transition temperature where long-range order has disappeared. In this regime the mean-field component of the potential vanishes and the potential function reduces to that of isolated interacting pairs. A theory of isolated interacting pairs is, of course, incapable of modeling a highly interacting cooperative system.

The theory presented in this paper has been based on a model in which the interaction potentials are assumed to be volume (temperature) independent (constant $v_{0}$ ). An immediate consequence of this restriction is the absence in the theory of the small experimentally observed volume discontinuity at the phase transition. The absence of the volume discontinuity then influences the magnitude of the theoretical latent heats and entropies of transition. In addition, the neglect of the volume (temperature) dependence of the interactions influences the precise temperature dependence of the order parameters and thermodynamic functions. Thus, though our calculations are in reasonable agreement with experiment, a thorough and more realistic fitting of experimental data will require the inclusion of the volume (temperature) dependence of the interactions potentials in the formal development of the theory.

\section{APPENDIX}

Here we show that at any particular temperature the value of $\beta F$ given by Eq. (15), with the $\sigma_{L}$ determined from Eq. (12), is unique up to a constant. 


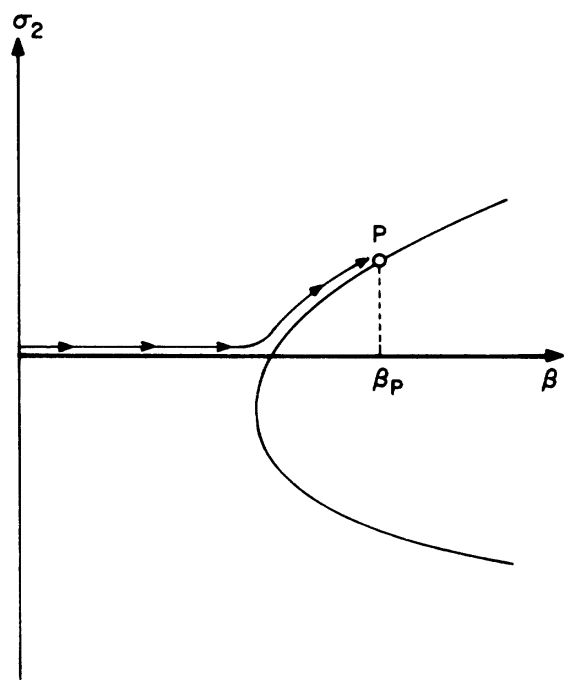

FIG. 13. Schematic representation of the loci of the solutions to the self-consistency condition, Eq. (12). The arrows indicate the path of integration in Eq. (A1).

Figure 13 shows a schematic drawing of the loci of the solutions to the self-consistency relation, Eq. (12). For simplicity of illustration we have only shown the loci in the $\sigma_{2}-\beta$ plane. However, the arguments presented in the following are equally applicable to the general case. We note in Fig. 13 that $\sigma_{2}=0$ is a solution at all temperatures and that two more branches of solutions appear only below a certain temperature. The freeenergy function, $\beta F$, can now be defined on the $\sigma_{2}-\beta$ plane as a surface whose distance above or below the $\sigma_{2}-\beta$ plane at any particular point gives the value of $\beta F$ at that point. On this free-energy surface there is a trough formed by the local minima of the free energy. The position of the bottom of the trough is traced out by the loci on the $\sigma_{2}-\beta$ plane as shown in Fig. 13. Suppose now we want the value of $\beta F$ at the point $P$. Let the value of $\beta F$ at $\beta=0, \sigma_{2}=0$ be a constant $C$. The value of $\beta F$ at $P$ is then given by

$$
\beta F(\text { at } P)=C+\int_{0}^{p}\left(\frac{\partial(\beta F)}{\partial \sigma_{2}} \hat{\sigma}_{2}+\frac{\partial(\beta F)}{\partial \beta} \hat{\beta}\right) \cdot d \overrightarrow{\mathrm{S}}
$$

where the integral is along the path indicated by arrows in Fig. 13. $\hat{\sigma}_{2}, \hat{\beta}$ denote unit vectors along the $\sigma_{2}$ and $\beta$ axes, respectively, and $d \overrightarrow{\mathrm{S}}$ is a line element along the path of integration: $\hat{\beta} \cdot d \overrightarrow{\mathrm{S}}=d \beta$, $\hat{\sigma}_{2} \cdot d \overrightarrow{\mathrm{S}}=d \sigma_{L}$. Since on the path of integration,

$$
\frac{\partial(\beta F)}{\partial \sigma_{2}}=0, \frac{\partial(\beta F)}{\partial \beta}=E,
$$

as discussed in Sec. IIC, it immediately follows that

$$
\beta F(\text { at } P)=C+\int_{0}^{\beta} P\left[\sigma_{L}^{0}(\beta), \beta\right] d \beta .
$$

Here $\beta_{P}$ is the value of $\beta$ at point $P$ as shown in Fig. 13, and $\sigma_{L}^{0}(\beta)$ denotes the values of the $\sigma_{L}$ determined by Eq. (12) $\left[\sigma_{2}^{0}(\beta)\right.$ gives the loci on the $\sigma_{2}-\beta$ plane as shown in Fig. 13]. Since the value of $E$ is well defined at every point of the loci, Eq. (A3) shows that up to a constant, the two conditions as given by Eq. (A2) uniquely determine the values of $\beta F$ on the loci. Since the function $\beta F$ as given by Eq. (15) satisfies these two conditions, this proves our assertion.

Note that we have only proven the uniqueness of the $\beta F$ values on the loci of the solutions to Eq. (12). Indeed, different functions of $\beta F$ may be found which have identical values on the loci [and therefore satisfy the two conditions given by Eq. (A2)] but differ elsewhere on the $\sigma_{2}-\beta$ plane.
${ }^{1}$ L. Onsager, Ann. N. Y. Acad. Sci. 51, 627 (1949).

${ }^{2}$ P. Sheng, RCA Rev. 35, 132 (1974).

${ }^{3}$ W. Maier and A. Saupe, Z. Narturforsch. A 14,882 (1959); 15, 287 (1960).

${ }^{4}$ P. J. Wojtowicz, RCA Rev. 35, 105 (1974).

${ }^{5}$ S. Chandrasekhar and N. V. Madhusudana, Acta Crystallogr. A 27, 303 (1971).

${ }^{6}$ R. L. Humphries, P. G. James, and G. R. Luckhurst, J. Chem. Soc. Faraday Trans. II 68, 1031 (1972).

${ }^{7}$ P. J. Wojtowicz, RCA Rev. 35, $11 \overline{8}$ (1974).

${ }^{8}$ G. Lasher, Phys. Rev. A $5, \overline{13} 50$ (1972).

${ }^{9}$ P. A. Lebwohl and G. Lasher, Phys. Rev. A $\underline{6}, 426$ (1972); 7, 2222 (1973).

${ }^{10} \mathrm{P}$. W. Kasteleijn and J. Van Kronendonk, Physica (Utr.) 22, 317 (1956).

${ }^{11}$ B. Strieb, H. B. Callen, and G. Horwitz, Phys. Rev. 130,1798 (1963).
${ }^{12}$ R. J. Elliot, J. Phys. Chem. Solids 16, 165 (1960).

${ }^{13}$ H. B. Callen and E. Callen, Phys. Rev. 136, A1675 (1964).

${ }^{14}$ H. B. Callen and E. Callen, J. Phys. Soc. Jpn. 20, 1980 (1965).

${ }^{15}$ E. Callen and H. B. Callen, J. Appl. Phys. 36, 1140 (1965).

${ }^{16} \mathrm{~J}$. S. Smart, Effective Field Theories of Magnetism (Saunders, Philadelphia, 1966), Chap. 5.

${ }^{17}$ J. C. Raich, R. D. Etters, and L. Flax, Chem. Phys. Lett. 6, 491 (1970).

${ }^{18}$ R. G. Priest, Phys. Rev. Lett. 26, 423 (1971).

${ }^{19}$ J. A. Pople, Proc. R. Soc. A $2 \overline{21}, 498$ (1954).

${ }^{20} \mathrm{M}$. E. Rose, Elementary Theory of Angular Momentum (Wiley, New York, 1957).

${ }^{21}$ T. Oguchi, Prog. Theor. Phys. 13, 148 (1955).

${ }^{22}$ N. V. Madhusudana and S. Chandrasekhar, Solid State 
Commun. 13, 377 (1973).

${ }^{23}$ T. D. Schultz, Mol. Crystallogr. Liq. Crystallogr. 14, 147 (1971).

${ }^{24}$ E. B. Priestley, P. J. Wojtowicz, and P. Sheng, Introduction to Liquid Crystals (Plenum, New York, 1975), p. 351 .
${ }^{25}$ Data of S. H. Glarum and J. H. Marshall, J. Chem. Phys. 44, 2884 (1966); A. Saupe, Angew. Chem. (Inter. Ed.) 7, 97 (1968); S. Chandrasekhar and N. V. Madhusudana, J. Phys. C 30, 24 (1969); as quoted in Ref. 5. ${ }^{26}$ Data of H. Arnold, Z. Phys. Chem. 226, 146 (1964), as quoted in Ref. 5. 